\title{
Random Walk Radiosity with generalized absorption probabilities
}

\author{
Mateu Sbert \\ Institut d'Informàtica i Aplicacions \\ Universitat de Girona \\ mateu@ima.udg.es
}

\begin{abstract}
In this paper we study random walk estimators for radiosity with generalized absorption probabilities. That is, a path will either die or survive on a patch according to an arbitrary probability. The estimators studied so far, the infinite path length estimator and finite path length one, can be considered as particular cases. Practical applications of the random walks with generalized probabilities are given. A necessary and sufficient condition for the existence of the variance is given, together with an heuristics to be used in practical cases. The optimal probabilities are also found for the case when we are interested in the whole scene, and are equal to the reflectivities.
\end{abstract}

Keywords: Radiosity, Monte Carlo, Random Walk, Variance.

\section{Introduction}

Discrete or continuous random walk estimators have been widely used in radiosity. Gathering random walk proceeds sending paths from the patches of interest to gather energy when a source is hit. Path-tracing [6], and even distributed ray-tracing $[3,21]$ can be considered as the limiting case of gathering random walk for the non-discrete case (without the shadow ray). Shooting random walk shoots paths carrying energy from the sources, to update the visited patches [9], [2]. The techniques in [17, 5] can be seen as a breadth-first approach to a shooting random walk estimator, which in turn would be the depth-first approach. Bidirectional ray-tracing $[20,7]$ is a mixture of non-discrete shooting and gathering. The random walk proceeds according to the discrete Form Factor probability transitions. The survival (or not absorption) probability on a patch has been usually considered equal to its reflectivity. An exception to this survival probability is found in [8], where the received importance was considered instead of the reflectivity. In [10] we find also a short discussion under the term of survival biasing. Also, infinite path length estimators can be considered the ones where the survival probability is equal to one. We will study in this paper shooting and gathering estimators resulting of considering any survival (or not absorption) probability. We will keep the Form Factors as transition probabilities. In this way the finite path length estimators [12] and infinite ones [14] studied so far will be considered as particular cases of this generalized one.

The organization of this paper is as follows: In section 2 we present our previous work on random walk. In section 3 we study the gathering estimator with generalized absorption probabilities. A necessary and sufficient conditions for the existence of the variances is given, together with a heuristics to be used in practical situations. We give also an example of when to use this estimator. Next, in section 4, the shooting estimator is studied. The optimal survive probabilities for the case when we are interested in the whole scene are given. The resulting estimator happens to be the one with survival probability equal to the reflectivity. Finally, in section 5 we present our conclusions and future research.

\section{Previous Work}

In [12] we studied the three estimators defined in [18], together with their gathering dual ones. In [14] we characterized the infinite path length estimators, and in [16] we proved that the best finite path length estimator was better than the biased infinite path length one. Finally, in [15] we obtained the variances for the previous shooting estimators for any general source selection probability, and also proved that the results obtained so far were extensible to the pure particle tracing case, that is, when we keep the impinging point on a patch as next exiting point. The obtained results are summarized in table 1 .

A feature common to all the studied estimators is that we use as transition probabilities the Form Factors, and as survive (or not absorption) probability the reflectivity of the patch (except of course for the infinite path length, where the survival probability is always 1). Here we will relax this second assumption, that is, we will consider generalized absorption probabilities. The two already studied cases, when 
Table 1. Different Random Walk estimators. The meaning of the different quantities is in table 2.

\begin{tabular}{|c|c|c|}
\hline Shooting & Patch scored & Variance \\
\hline$\frac{\Phi_{T}}{1-R_{i}}$ & last & $\sum_{s} \Phi_{s} \frac{R_{i} b_{i s}}{A_{i} p_{s}\left(1-R_{i}\right)}-b_{i}^{2}$ \\
\hline$\frac{\Phi_{T}}{R_{i}}$ & all but last & $\sum_{s} \Phi_{s} \frac{R_{i} b_{i s}}{A_{i} p_{s}}\left(\frac{1}{R_{i}}+2 \xi_{i}\right)-b_{i}^{2}$ \\
\hline$\Phi_{T}$ & all & $\sum_{s} \Phi_{s} \frac{R_{i}\left(1+2 R_{i} \xi_{i}\right) b_{i s}}{A_{i} p_{s}}-b_{i}^{2}$ \\
\hline infinite & all & $\sum_{s} \Phi_{s} \frac{\left(1+2 R_{i} \xi_{i}\right) \beta_{i s}}{A_{i} p_{s}}-b_{i}{ }^{2}$ \\
\hline Gathering & Patch scored & Variance \\
\hline$\frac{E_{s}}{1-R_{s}}$ & last & $\frac{R_{i}}{p_{i}} \sum_{s} \frac{E_{s}}{\left(1-R_{s}\right)} b_{i s}-b_{i}{ }^{2}$ \\
\hline$\frac{E_{s}}{R_{s}}$ & all but last & $\frac{R_{i}}{p_{i}} \sum_{s} \frac{E_{s}+2 b_{s}}{R_{s}} b_{i s}-b_{i}{ }^{2}$ \\
\hline$E_{s}$ & all & $\frac{R_{i}}{p_{i}} \sum_{s}\left(E_{s}+2 b_{s}\right) b_{i s}-b_{i}{ }^{2}$ \\
\hline infinite & all & $\frac{1}{p_{i}} \sum_{s}\left(E_{s}+2 b_{s}\right) \beta_{i s}-b_{i}{ }^{2}$ \\
\hline
\end{tabular}

survival probability is equal to reflectivity and the infinite case, can then be seen as particular cases. We will still consider the Form Factors as transition probabilities. The usefulness of the estimators obtained can be seen when one considers survival probability proportional to importance (or better to received importance). This has been used in [8]. We will also restrict ourselves to the estimators that score on all patches. The extension of the results to the other ones, which score only on surviving patches or on the last patch, is straigthforward. Next we will proceed to study the gathering case.

\section{A gathering estimator with generalized ab- sorption probabilities}

We will consider here the discrete random walk, that is, the one which proceeds according to patch-to-patch Form Factors. However, the formulae and results obtained are also valid for the point-to-point Form Factors, as shown in [15].

Let us first consider what the expected value of any unbiased Monte Carlo estimator should be for the radiosity of a patch. Let us suppose that the emittance of source $s$ is $E_{s}, b_{i}$ is the reflected radiosity, or radiosity of patch $i$ due to the received power (that is, $b_{i}=B_{i}-E_{i}$, and so for a non-emitter patch, it equals the total radiosity), $F_{k l}$ denotes the Form Factor from patch $k$ to patch $l$, and $R_{k}$ denotes the reflectance of patch $k$. Then we have, by developing the Radiosity system in Neumann series (dropping the zero order
Table 2. Meaning of the different quantities appearing in table 1 . The suffix $i$ means for patch $i$, suffix $s$ indexes the sources.

\begin{tabular}{|c|c|}
\hline$E_{i}$ & Emissivity \\
\hline$b_{i}$ & Reflected radiosity $=B_{i}-E_{i}$ \\
\hline$\beta_{i}$ & idem with each reflectivity substituted by its square \\
\hline$\Phi_{s}$ & Area \\
\hline$A_{i}$ & Reflectivity \\
\hline$R_{i}$ & Reflected radiosity on $i$ due to source $s$ \\
\hline$\xi_{i}$ & $\begin{array}{c}\text { Received power (or radiosity) due to self- } \\
\text { emitted unit power (or emittance) }\end{array}$ \\
\hline$b_{i s}$ & Refled substituted by its square \\
\hline$\beta_{i s}$ & idem with each reflectivity substity for a path to begin at $i$ \\
\hline$p_{i}$ & Probabilit \\
\hline
\end{tabular}

term):

$$
\begin{aligned}
b_{i}=R_{i} & \sum_{s} E_{s} F_{i s}+R_{i} \sum_{h} \sum_{s} E_{s} F_{i h} R_{h} F_{h s} \\
& +R_{i} \sum_{h} \sum_{j} \sum_{s} E_{s} F_{i h} R_{h} F_{h j} R_{j} F_{j s}+\cdots
\end{aligned}
$$

This can be expressed as:

$$
b_{i}=b_{i}^{(1)}+b_{i}^{(2)}+b_{i}^{(3)}+\cdots
$$

where

$b_{i}^{(1)}=R_{i} \sum_{s} E_{s} F_{i s}, b_{i}^{(2)}=R_{i} \sum_{s} \sum_{h} E_{s} F_{i h} R_{h} F_{h s}$,

$b_{i}^{(3)}=R_{i} \sum_{s} \sum_{h} \sum_{j} E_{s} F_{i h} R_{h} F_{h j} R_{j} F_{j s}$ and so on. That is, $b_{i}^{(1)}$ represents the radiosity due to direct illumination, $b_{i}^{(2)}$ represents the radiosity after one bounce, and so on. It is also useful to define the following quantities:

$$
b_{i s}=b_{i s}^{(1)}+b_{i s}^{(2)}+\cdots
$$

$b_{i s}^{(1)}$ represents the radiosity due to direct illumination from source $s, b_{i s}^{(2)}$ represents the radiosity after one bounce from source $s$, and so on. It is clear that:

$$
b_{i}=\sum_{s} b_{i s}
$$

Let us now consider the following simulation. A path $\gamma$ starts from patch $i$ with probability $p_{i}$ (this probability can be considered as the initial or emitted importance of the patch), and from here on it evolves according to the transition probabilities given by the Form Factors. For instance, 
from $i$ it will go to patch $j$ with probability $F_{i j}$. It will then be absorbed in patch $j$ with probability $1-\theta_{j}$, and survive with probability $\theta_{j}$. Let us define now the random variables $\widehat{b}_{i}^{(1)}, \widehat{b}_{i}^{(2)}, \widehat{b}_{i}^{(3)}, \ldots$ in the following way:

All of those random variables are initially null. If the path $\gamma$ happens to arrive at source $s$ at length $l$, and if $i, h_{1}, h_{2}, \ldots, h_{l-1}, s$ is the trajectory the path has followed, then the value of $\widehat{b}_{i}^{(l)}$ is set to $R_{i} \frac{R_{h_{1}}}{\theta_{h_{1}}} \frac{R_{h_{2}}}{\theta_{h_{2}}} \ldots \frac{R_{h_{l-1}}}{\theta_{h_{l-1}}} \frac{E_{s}}{p_{i}}$. Let us define also a new random variable $\widehat{b}_{i}$ as:

$$
\widehat{b}_{i}=\widehat{b}_{i}^{(1)}+\widehat{b}_{i}^{(2)}+\widehat{b}_{i}^{(3)}+\cdots
$$

Now let us find the expected value of those random variables. Applying the definition of expected value, and remembering that the probability of selecting patch $i$ is $p_{i}$, the probability of landing on source $s$ just after leaving patch $i$ is $F_{i s}$, we have

$$
E\left(\widehat{b}_{i}^{(1)}\right)=\sum_{s} R_{i} \frac{E_{s}}{p_{i}} \times p_{i} F_{i s}=b_{i}^{(1)}
$$

Now, to go from patch $i$ to a source $s$ in a two length path we can pass through any patch $h$ (after surviving on it with probability $\theta_{h}$, so we have

$$
\begin{aligned}
E\left(\widehat{b}_{i}^{(2)}\right) & =\sum_{h} \sum_{s} R_{i} \frac{R_{h}}{\theta_{h}} \frac{E_{s}}{p_{i}} \times p_{i} F_{i h} \theta_{h} F_{h s} \\
& =b_{i}^{(2)}
\end{aligned}
$$

and so on. Then, we have

$$
\begin{aligned}
E\left(\widehat{b}_{i}\right) & =E\left(\widehat{b}_{i}^{(1)}+\widehat{b}_{i}^{(2)}+\cdots\right)=E\left(\widehat{b}_{i}^{(1)}\right)+E\left(\widehat{b}_{i}^{(2)}\right)+\cdots \\
& =b_{i}^{(1)}+b_{i}^{(2)}+\cdots=b_{i}
\end{aligned}
$$

So it is clear that the random variable $\widehat{b}_{i}^{(l)}$ is a centered estimator for the radiosity due to the power arrived on patch $i$ after $l$ bounces, and the sum of all this family of estimators gives a new centered estimator $\widehat{b}_{i}$ which corresponds to the total radiosity of patch $i$ due to the power arrived after any number of bounces. Our aim now is to obtain the variance for this estimator. We will use here a similar approach to the one in [12] and [14]. We can decompose $\operatorname{Var}\left(\widehat{b}_{i}\right)$ in the following way

$$
\begin{aligned}
\operatorname{Var}\left(\widehat{b}_{i}\right) & =\operatorname{Var}\left(\widehat{b}_{i}^{(1)}+\widehat{b}_{i}^{(2)}+\cdots\right) \\
& =E\left(\left(\widehat{b}_{i}^{(1)}+\widehat{b}_{i}^{(2)}+\cdots\right)^{2}\right)-\left(E\left(\widehat{b}_{i}\right)\right)^{2} \\
& =E\left(\widehat{b}_{i}^{(1) 2}\right)+E\left(\widehat{b}_{i}^{(2) 2}\right)+\cdots \\
& +2 \sum_{1 \leq n<m} E\left(\widehat{b}_{i}^{(n)} \widehat{b}_{i}^{(m)}\right)-b_{i}{ }^{2}
\end{aligned}
$$

The terms of the form $E\left(\widehat{b}_{i}^{(n)} \widehat{b}_{i}^{(m)}\right)$ are not null, because if a path arrives at length $n$ on source $s$ it can also arrive later at source $s^{\prime}$ at length $m$. Next we find them:

$$
\begin{aligned}
& E\left(\widehat{b}_{i}^{(n)} \widehat{b}_{i}^{(m)}\right) \\
= & \sum_{s} \sum_{s^{\prime}} \sum_{h_{1}} \cdots \sum_{h_{n-1}} \sum_{h_{n+1}} \cdots \sum_{h_{m-1}} R_{i} \frac{R_{h_{1}}}{\theta_{h_{1}}} \cdots \frac{R_{h_{n-1}}}{\theta_{h_{n-1}}} \frac{E_{s}}{p_{i}} . \\
& R_{i} \frac{R_{h_{1}}}{\theta_{h_{1}}} \cdots \frac{R_{h_{n-1}}}{\theta_{h_{n-1}}} \frac{R_{s}}{\theta_{s}} \frac{R_{h_{n+1}}}{\theta_{h_{n+1}}} \cdots \frac{R_{h_{m-1}}}{\theta_{h_{m-1}}} \frac{E_{s^{\prime}}}{p_{i}} . \\
& p_{i} F_{i h_{1}} \theta_{h_{1}} \cdots F_{h_{n-1} s} \theta_{s} F_{s h_{n+1}} \theta_{h_{n+1}} \cdots F_{h_{m-1} s^{\prime}} \\
= & \theta_{i} \sum_{s} \sum_{h_{1}} \cdots \sum_{h_{n-1}} \frac{R_{i}^{2}}{\theta_{i}} \frac{R_{h_{1}}^{2}}{\theta_{h_{1}}} \cdots \frac{R_{h_{n-1}}^{2}}{\theta_{h_{n-1}}} \frac{E_{s}}{p_{i}} . \\
& \sum_{i} \sum_{s} \sum_{h_{h_{1}+1}} \cdots \sum_{h_{h_{n-1} s} s} \cdot R_{s} F_{s h_{n+1}} R_{h_{n+1}} \cdots R_{h_{m-1}} F_{h_{m-1} s^{\prime}} \frac{E_{s^{\prime}}}{p_{i}} \\
= & \frac{\theta_{i}}{p_{i}} \sum_{s} \epsilon_{i s}^{(n)} \sum_{s^{\prime}} b_{s s^{\prime}}^{(m-n)}=\frac{\theta_{i}}{p_{i}} \sum_{s} \epsilon_{i s}^{(n)} b_{s}^{(m-n)}
\end{aligned}
$$

where $\epsilon_{i s}^{(n)}$ is the radiosity due to the incoming energy after $n$ bounces in the same environment having changed all the reflectivities by their square divided by $\theta_{i}$, that is, $R_{i}$ is substituted by $\frac{R_{i}^{2}}{\theta_{i}}$. Then

$$
\begin{aligned}
\sum_{1 \leq n}\left(\sum_{n<m} E\left(\widehat{b}_{i}^{(n)} \widehat{b}_{i}^{(m)}\right)\right) & =\frac{\theta_{i}}{p_{i}} \sum_{s} \sum_{1 \leq n} \epsilon_{i s}^{(n)} \sum_{n<m} b_{s}^{(m-n)} \\
& =\frac{\theta_{i}}{p_{i}} \sum_{s} \epsilon_{i s} b_{s}
\end{aligned}
$$

and also

$$
\begin{aligned}
E\left(\widehat{b}_{i}^{(1) 2}\right) & =\sum_{s}\left(R_{i} \frac{E_{s}}{p_{i}}\right)^{2} \times p_{i} F_{i s} \\
& =\sum_{s} \frac{\theta_{i} E_{s}}{p_{i}} \frac{R_{i}^{2}}{\theta_{i}} F_{i s} E_{s} \\
& =\frac{\theta_{i}}{p_{i}} \sum_{s} E_{s} \epsilon_{i s}^{(1)} \\
E\left(\widehat{b}_{i}^{(2) 2}\right)= & \sum_{h} \sum_{s}\left(R_{i} \frac{R_{h}}{\theta_{h}} \frac{E_{s}}{p_{i}}\right)^{2} \times p_{i} F_{i h} \theta_{h} F_{h s} \\
= & \frac{\theta_{i}}{p_{i}} \sum_{s} E_{s} \epsilon_{i s}^{(2)}
\end{aligned}
$$

and so on. Then we obtain

$$
\begin{aligned}
\operatorname{Var}\left(\widehat{b}_{i}\right) & =\frac{\theta_{i}}{p_{i}} \sum_{s} E_{s}\left(\epsilon_{i s}^{(1)}+\epsilon_{i s}^{(2)}+\cdots\right) \\
& +2 \frac{\theta_{i}}{p_{i}} \sum_{s} \epsilon_{i s} b_{s}-b_{i}{ }^{2} \\
& =\frac{\theta_{i}}{p_{i}} \sum_{s}\left(E_{s}+2 b_{s}\right) \epsilon_{i s}-b_{i}{ }^{2}
\end{aligned}
$$


where $\epsilon_{i s}$ is the radiosity due to the incoming energy in the same environment having changed all the reflectivities by their square divided by the survival (or not absorption) probability. For the radiosity our estimator is simply $\widehat{b}_{i}+E_{i}$, and as $E_{i}$ is a constant we have

$$
\operatorname{Var}\left(\widehat{b}_{i}+E_{i}\right)=\frac{\theta_{i}}{p_{i}} \sum_{s}\left(E_{s}+2 b_{s}\right) \epsilon_{i s}-b_{i}{ }^{2}
$$

\subsection{Existence of the variance}

We have that $\epsilon_{i}$ are the reflected radiosities solution of the same system as the original one, having substituted each reflectivity $R_{i}$ by $\frac{R_{i}^{2}}{\theta_{i}}$. But for these quantities to exist (and be positive !) we must have as necessary and sufficient condition that the spectral radius be less than 1 [19], that is

$$
\rho\left(\frac{R_{i}^{2}}{\theta_{i}} F_{i j}\right)<1
$$

This condition is equivalent to the one given in [4] for the existence of variance in a random walk, that is

$$
\lim _{k \rightarrow \infty}\left(\operatorname{norm}\left(U^{k}\right)\right)^{\left(\frac{1}{k}\right)}<1
$$

where $U_{i j}=\frac{K_{i j}^{2}}{p_{i j}}, K_{i j}$ is the kernel and $p_{i j}$ are the transition probabilities (which include the survival ones). In our case $U_{i j}=\frac{R_{i}^{2} F_{i j}^{2}}{\theta_{i} F_{i j}}=\frac{R_{i}^{2} F_{i j}}{\theta_{i}}$.

The condition (3) is satisfied if for all $i \frac{R_{i}^{2}}{\theta_{i}}<1$, or $\theta_{i}>R_{i}^{2}$. To fulfill this condition, if we are initially given survival probabilities $\theta_{i}$, we just substitute them by $\max \left(\theta_{i}, R_{i}^{2}+\delta\right)$, where $\delta$ can be taken as small as we want (and of course, $R_{i}^{2}+\delta$ must be less than 1 ).

In fact, what does the constraint $\frac{R_{i}^{2}}{\theta_{i}}<1$ is to ensure the convergence of the infinite sums in formula (1). We are not claiming that without this condition there can not be convergence. What we are claiming is that, to be safe, we should take care of this condition. And of course we can show that if for all patches this condition is violated, there is no convergence. Next we prove that $\lim _{n \rightarrow \infty} E\left(\widehat{b}_{i}^{(n) 2}\right) \neq 0$, and thus the sum can not converge.

$$
\begin{aligned}
E\left(\widehat{b}_{i}^{(n) 2}\right)= & \sum_{h_{1}} \cdots \sum_{h_{n-1}} \sum_{s}\left(R_{i} \frac{R_{h_{1}}}{\theta_{h_{1}}} \ldots \frac{R_{h_{n-1}}}{\theta_{h_{n-1}}} \frac{E_{s}}{p_{i}}\right)^{2} \\
& p_{i} F_{i_{h_{1}}} \theta_{h_{1}} \ldots F_{i_{h_{n-1}}} \theta_{h_{n-1}} F_{h s} \\
= & \sum_{h_{1}} \cdots \sum_{h_{n-1}} \sum_{s} R_{i}^{2} \frac{R_{h_{1}}^{2}}{\theta_{h_{1}}} \ldots \frac{R_{h_{n-1}}^{2}}{\theta_{h_{n-1}}} \frac{E_{s}^{2}}{p_{i}} \\
& F_{i_{h_{1}}} \ldots F_{i_{h_{n-1}}} F_{h s} \\
\geq & \frac{R_{i}^{2}}{p_{i}} \sum_{h_{1}} \cdots \sum_{h_{n-1}} \sum_{s} F_{i_{h_{1}}} \ldots F_{i_{h_{n-1}}} F_{h s} E_{s}^{2}
\end{aligned}
$$

because we have supposed for all $i \frac{R_{i}^{2}}{\theta_{i}} \geq 1$. But we have

$$
\sum_{h_{1}} \cdots \sum_{h_{n-1}} F_{i_{h_{1}}} \ldots F_{i_{h_{n-1}}} F_{h s}=\left(F^{n}\right)_{i s}
$$

and in [13] we prove that, whenever the Form Factor matrix is irreducible and aperiodic

$$
\lim _{n \rightarrow \infty}\left(F^{n}\right)_{i s}=\frac{A_{s}}{A_{T}}
$$

where $A_{T}$ is the total area in the scene. We do not consider geometrically meaningful for a Form Factor matrix to be periodic. If there are closed rooms, it is reducible with one submatrix for each room. In this case, we consider in turn each room and we have an irreducible matrix.

But now from (4) and (5) we obtain:

$$
\begin{aligned}
\lim _{n \rightarrow \infty} E\left(\widehat{b}_{i}^{(n) 2}\right) & \geq \sum_{s}\left(\lim _{n \rightarrow \infty}\left(F^{n}\right)_{i s}\right) E_{s}^{2} \\
& =\sum_{s} \frac{A_{s}}{A_{T}} E_{s}^{2}>0
\end{aligned}
$$

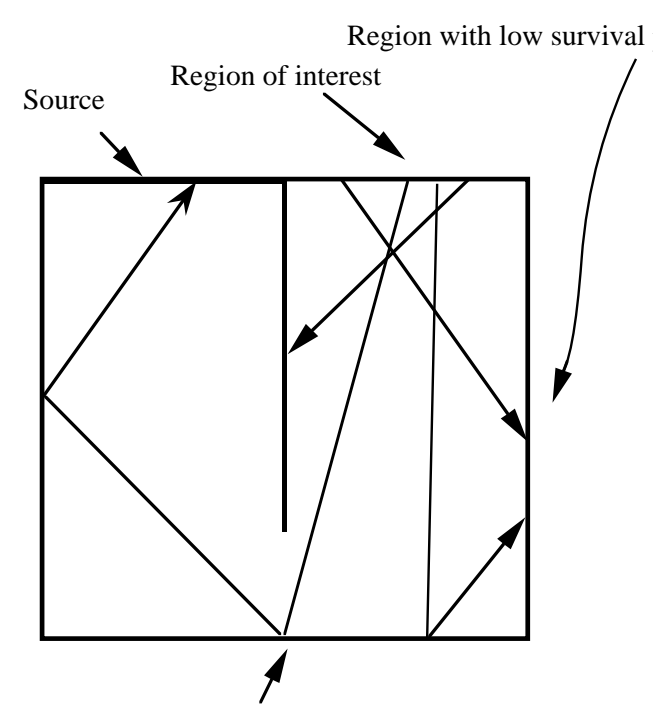

Region with higher survival probability

\section{Figure 1. Paths traced from the region of inter- est have a higher survival probability where the reflected radiosity is higher.}

As the condition $\theta_{i}>R_{i}^{2}$ for the existence of the variance is too restrictive, the question arises whether a weaker condition can be given. We have run simulations and found that the spectral radius (3) is approximated by, and in most cases, is less than, the average value

$$
\left(\frac{R^{2}}{\theta}\right)_{\text {ave }}=\frac{1}{A_{T}} \sum A_{i} \frac{R_{i}^{2}}{\theta_{i}}
$$


This finding is similar to the results in [1]. It is based on the following:

If we consider the series $\rho\left(\frac{R_{i}^{2}}{\theta_{i}}\left(F^{n}\right)_{i j}\right)$, its limit is $\rho\left(\frac{R_{i}^{2}}{\theta_{i}} \frac{A_{i}}{A_{T}}\right)$, but it is very easy to check that

$$
\rho\left(\frac{R_{i}^{2}}{\theta_{i}} \frac{A_{i}}{A_{T}}\right)=\frac{1}{A_{T}} \sum_{i} A_{i} \frac{R_{i}^{2}}{\theta_{i}}=\left(\frac{R^{2}}{\theta}\right)_{a v e}
$$

Thus what we do is to approximate the first term in the series, $\rho\left(\frac{R_{i}^{2}}{\theta_{i}} F_{i j}\right)$, by the limit of the series.

This approximation will work as far as we do not have almost isolated rooms with very different reflectivities (we can not have completely isolated rooms, as we only consider here an irreducible Form Factor matrix). Excluding those cases, a heuristics such as keeping this average value less than 0.8 or maybe 0.9 should be safe.

\subsection{Particular cases}

When $\theta_{i}=1$ we have the infinite path length estimator, $\frac{R_{i}^{2}}{\theta_{i}}=R_{i}^{2}$ and $\epsilon_{i s}$ becomes $\beta_{i s}$, and we obtain again the formula in section 2. On the other hand, when $\theta_{i}=R_{i}$ we have the $E_{s}$ estimator, $\frac{R_{i}^{2}}{\theta_{i}}=R_{i}$ and $\epsilon_{i s}$ becomes $b_{i s}$, obtaining again the formula for the $E_{s}$ estimator given in section 2 .

\subsection{An example}

Suppose we have obtained a coarse solution for the radiosities. This solution could be used to drive the random walk taking $\theta_{i} \propto B_{i}-E_{i}$. This will assure that the paths will survive in patches with high received radiosity. This is useful in a scene alike the one in Figure 1. This case can be considered the dual of the one given in [8].

\section{The shooting estimator}

Let us now consider the shooting estimator with generalized absorption probabilities. Consider the following simulation. A path $\gamma$ starts from source $s$ with probability $p_{s}$, and from here on it evolves according to the transition probabilities given by the Form Factors. For instance, from $s$ it will go to patch $j$ with probability $F_{s j}$. On each patch $i$ hit, a survival-absorption test is done according to the probabilities $\left\{\theta_{i}, 1-\theta_{i}\right\}$. If the path $\gamma$ happens to arrive to the patch $i$ at length $l$, then the radiosity of this patch is updated with the quantity $\frac{R_{i}}{p_{s}} \frac{R_{h_{1}}}{\theta_{h_{1}}} \frac{R_{h_{2}}}{\theta_{h_{2}}} \ldots \frac{R_{h_{l-1}}}{\theta_{h_{l-1}}} \Phi_{s}$.

Now, the variance can be found either using the same approach as in section 3, or taking into account that the shooting estimator can be defined as the dual of the gathering one [15]. The variance is found to be:

$$
\operatorname{Var}\left(\widehat{b}_{i}+E_{i}\right)=\theta_{i} \sum_{s} \Phi_{s} \frac{\left(1+2 R_{i} \xi_{i}\right) \epsilon_{i s}}{A_{i} p_{s}}-b_{i}^{2}
$$

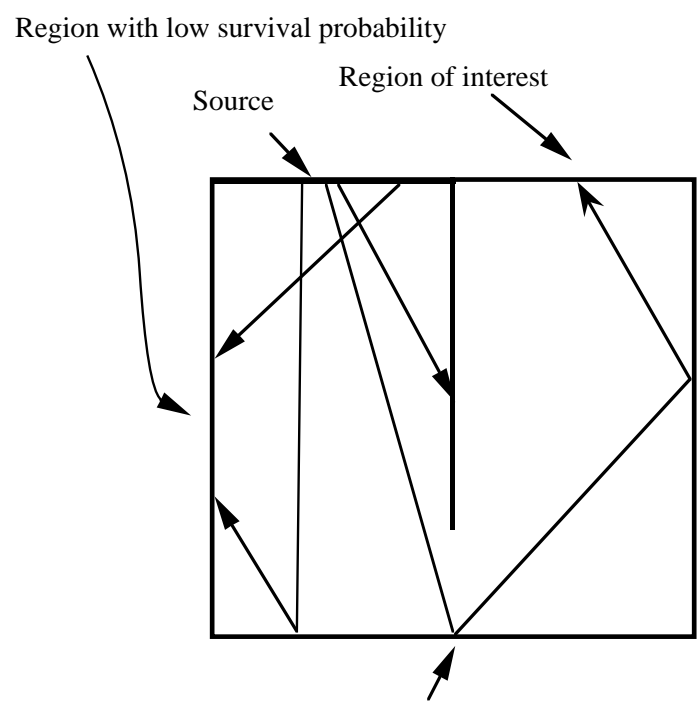

Region with higher survival probability

Figure 2. Paths traced from the source have a higher survival probability where the received importance towards the region of interest is higher.

Here is also valid the same argumentation as in previous section about the existence of the variance.

\subsection{Particular cases}

When $\theta_{i}=1$ we have the infinite path length estimator, $\frac{R_{i}^{2}}{\theta_{i}}=R_{i}^{2}$ and $\epsilon_{i s}$ becomes $\beta_{i s}$, and we obtain again the formula in section 2. On the other hand, when $\theta_{i}=R_{i}$ we have the $\Phi_{T}$ estimator, $\frac{R_{i}^{2}}{\theta_{i}}=R_{i}$ and $\epsilon_{i s}$ becomes $b_{i s}$, obtaining again the formula for the $\Phi_{T}$ estimator given in section 2 .

Formula (8), when taking source selection probabilities proportional to the power of the sources $p_{s}=\frac{\Phi_{s}}{\Phi_{T}}$ whith $\Phi_{T}$ total power, converts into

$$
\operatorname{Var}\left(\widehat{b}_{i}+E_{i}\right)=\theta_{i} \Phi_{T} \frac{\left(1+2 R_{i} \xi_{i}\right) \epsilon_{i}}{A_{i}}-b_{i}^{2}
$$

where $\epsilon_{i}=\sum_{s} \epsilon_{i s}$ is the reflected radiosity due to all sources changing $R_{i}$ by $\frac{R_{i}^{2}}{\theta_{i}}$. 


\subsection{An example}

If we make the survival probability proportional to received importance, that is, $\theta_{i} \propto I_{i}-V_{i}$, where the $V_{i}$ is the initial and $I_{i}$ the total importance [8], we will assure that the paths will survive in patches which are important to the selected ones. This could be used to drive a random walk in a scene alike the one in Figure 2, the dual of Figure 1. This case was the one considered in [8], although there a breadth-first strategy was used, instead of the considered here, which is depth-first.

\subsection{Optimal survival probabilities}

Suppose we are interested in all patches, not just in a single region. We want to find out the optimal survival probabilities $\theta_{i}$ in the sense to maximize the efficiency. This can be defined as the inverse of the product of the Variance times the cost [11], for a single patch, or the average weighted variances (expected value of the Mean Square Error) times the cost, for the whole scene. This means, taking as average cost $\frac{1}{1-\theta_{\text {ave }}}$, minimizing the quantity

$$
E(M S E) \times \frac{1}{1-\theta_{\text {ave }}}
$$

Now, using the definition of the Mean Square, formula (9) for the variance (we consider a reasonable hipothesis $p_{s}=\frac{\Phi_{s}}{\Phi_{T}}$ when interested on the whole scene) and the approximation

$$
\epsilon_{i} \approx \frac{\frac{R_{i}^{2}}{\theta_{i}} \Phi_{T}}{A_{T}\left(1-\left(\frac{R^{2}}{\theta}\right)_{a v e}\right)}
$$

we obtain, following the same approach as in [16]:

$$
E(M S E) \approx \frac{\Phi_{T}^{2} R_{a v e}^{2}}{A_{T} A_{\text {ave }}\left(1-\left(\frac{R^{2}}{\theta}\right)_{\text {ave }}\right)}
$$

which substituted in (10) (after approximating $\left(\frac{R^{2}}{\theta}\right)_{\text {ave }}$ by $\left.\frac{R_{a v e}^{2}}{\theta_{a v e}}\right)$ gives as quantity to minimize:

$$
\frac{\Phi_{T}^{2} R_{\text {ave }}^{2}}{A_{T} A_{\text {ave }}\left(1-\left(\frac{R_{\text {ave }}^{2}}{\theta_{\text {ave }}}\right)\right.} \frac{1}{1-\theta_{\text {ave }}}
$$

The behaviour of this quantity escalated for $\frac{\Phi_{T}^{2} R_{a v e}^{2}}{A_{T} A_{a v e}}=1$ is shown in figure 3 , taking the values for $R_{\text {ave }}$ of $0.3,0.5$ and 0.8 , respectively.

The analytical solution is $\theta_{\text {ave }}=R_{\text {ave }}$. This will obviously happen when for all $i \theta_{i}=R_{i}$. Thus we can state the result:

Between all the unbiased shooting random walk estimators with generalised absorption probabilities and transition probabilities the Form Factors, the most efficient for

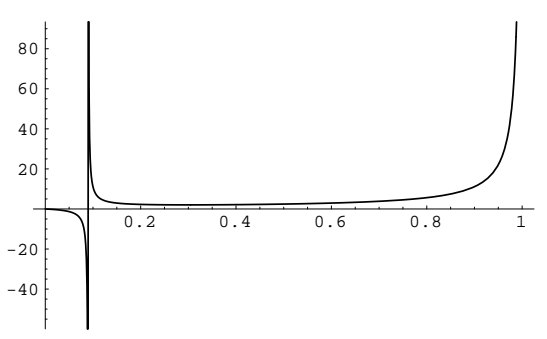

(a)

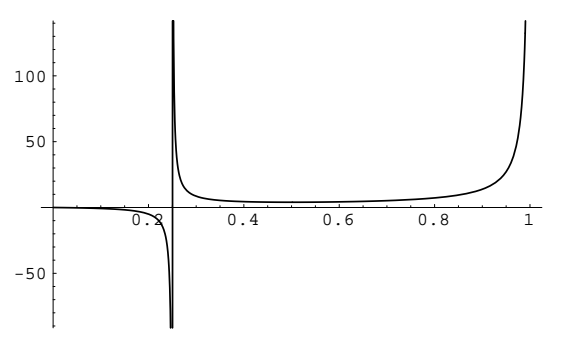

(b)

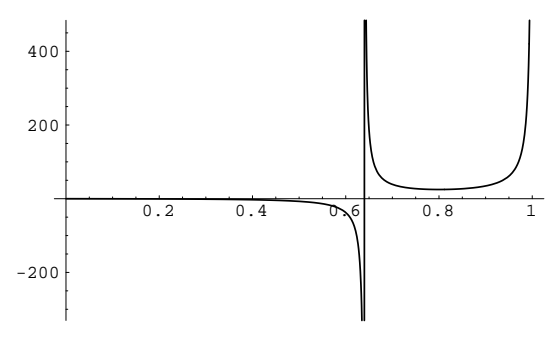

(c)

Figure 3. Behaviour of the inverse of efficiency against $\theta_{\text {ave }}$ for $R_{\text {ave }}=0.3,0.5$ and 0.8 , respectively. The vertical asymptote corresponds to $R_{a v e}^{2}$ 
calculating all radiosities is the one with survival probability equal to the reflectivity.

Remember that the infinite path length estimator is not considered here (the cost would be infinite), but from [16] we know that biasing it we obtain a much worse estimator than $\Phi_{T}$. That means that $\Phi_{T}$ is the best of all shooting random walk estimators with transition probabilities the Form Factors, biased or not. And as from [12] we know that shooting estimators are much better when dealing with the whole scene than the gathering ones, we can extend this result to all random walk estimators studied till now. It must be remembered here that the $\Phi_{T}$ estimator in its breadth-first approach was the one used by Shirley [17] and Feda and Purgathofer [5].

\section{Conclusions and future research}

We have here generalized the results of [12] and [14] to the case of a generalized absorption probability, obtaining closed formulae for the variances of the estimators studied. Those are presented in table 3 . A necessary and sufficient condition for the existence of the variance is also given, together with a heuristics to be used in practical cases. The usefulness of such estimators has also been shown. It has been proved that the best shooting estimator, when we are interested in the whole scene, comes from using the reflectivity as the survival probability. Future work will be directed towards obtaining closed formulae, whenever possible, for generalized transition probabilities.

\section{Table 3. Variances for Random Walk estima- tors with generalized absorption probabili- ties, $\theta_{i} . \epsilon_{i s}$ is the reflected radiosity in patch $i$ due to source $s$ substituting each reflectivity by $\frac{R_{i}}{\theta_{i}}$.}

\begin{tabular}{|c|c|}
\hline shooting & $\theta_{i} \sum_{s} \Phi_{s} \frac{\left(1+2 R_{i} \xi_{i}\right) \epsilon_{i s}}{A_{i} p_{s}}-b_{i}^{2}$ \\
\hline gathering & $\frac{\theta_{i}}{p_{i}} \sum_{s}\left(E_{s}+2 b_{s}\right) \epsilon_{i s}-b_{i}{ }^{2}$ \\
\hline
\end{tabular}

\section{Acknowledgements}

This project has been funded in part with grant number TIC 95-0614-C03-03 of the Spanish Government, grant number 1995SGR-00232 of CUR-Generalitat de Catalunya and Austrian-Spanish Acciones Integradas.

\section{References}

[1] Gladimir Baranoski, Randall Bramley and Peter Shirley, "Fast radiosity solutions for environments with high average reflectance", Sixth Eurographics Workshop on Rendering Appeared in Rendering Techniques '95 (eds. W. Purgathofer and P. Hanrahan), pp. 345-356, Springer, Wien, 1995.

[2] Philip Dutre and Yves D. Willems, "Importancedriven monte carlo light tracing", Fifth Eurographics Workshop on Rendering, pp. 185-194, Darmstadt, Germany, June 1994. Appeared in "Photorealistic Rendering Techniques" (eds. G. Sakas, P. Shirley, and S. Muller).

[3] R.L. Cook, T. Porter, L. Carpenter, "Distributed Ray Tracing", Computer Graphics (SIGGRAPH '84 Proc.), 18(3), pp. 137-145, July 1984.

[4] S.M. Ermakow, Die Monte-Carlo-Methode und verwandte Fragen. R. Oldenbourg Verlag, MünchenWien, 1975.

[5] Martin Feda and Werner Purgathofer, "Progressive ray refinement for monte carlo radiosity", Fourth Eurographics Workshop on Rendering, pp. 15-26, Paris, France, June 1993. Eurographics Technical Report Series EG 93 RW.

[6] J.T. Kajiya, "The Rendering Equation”, Computer Graphics (SIGGRAPH '86 Proc.), vol. 20, pp. 143150, August 1986.

[7] Eric P. Lafortune and Yves D. Willems, "Bidirectional path tracing", Proceedings of Third International Conference on Computational Graphics and Visualization Techniques (Compugraphics '93), pp. 145-153, Alvor, Portugal, December 1993.

[8] Attila Neumann, Laszlo Neumann, Philippe Bekaert, Yves Willems and Werner Purgathofer, "Importance-Driven Stochastic Ray Radiosity", Proceedings of the Seventh Eurographics Workshop on Rendering. Appeared in Rendering Techniques '96 (eds. X. Pueyo and P. Schroeder), pp. 111-122, 1996.

[9] S. N. Pattanaik and S. P. Mudur, "Computation of global illumination by monte carlo simulation of the particle model of light", Proceedings of the Third Eurographics Workshop on Rendering, pp. 71-83, Bristol, UK, May 1992.

[10] S. Pattanaik and S. Mudur, "Efficient potential equation solutions for global illumination computation", Computers \& Graphics, 17(4):387-396, 1993.

[11] Reuven Y. Rubinstein. Simulation and the Monte Carlo method. John Wiley \& Sons, New York, 1981.

[12] Mateu Sbert, "Error and complexity of random walk monte carlo radiosity", IEEE Transactions on Visualization and Computer Graphics, 3(1), March 1997.

[13] Mateu Sbert. The use of global random directions to compute radiosity. Global Monte Carlo methods. Ph.D. thesis, Universitat Politècnica de Catalunya, Barcelona, March 1997. Available in http://ima.udg.es/ $\sim$ mateu

[14] Mateu Sbert, "Variance of two infinite path length random walk radiosity estimators", Proceedings of WSCC'97, pp. 475-483, Plzen, February 1997. An extended version to appear in Computer\&Graphics. 
[15] Mateu Sbert, "Optimal source selection in shooting random walk radiosity", Proceedings of Eurographics'97,pp 301-308, Budapest, September 1997.

[16] Mateu Sbert, Alex Brusi "Comparing finite and biased infinite path length shooting random walk estimators for radiosity", Proceedings of SCCG'97, Budmerice, Slovakia, June 1997.

[17] Peter Shirley, "A ray tracing method for illumination calculation in diffuse-specular scenes", Proceedings of Graphics Interface '90, pp. 205-212, Toronto, Ontario, May 1990.

[18] P. Shirley, "Time Complexity of Monte Carlo Radiosity", Proceedings of Eurographics'91, Vienna, pp. 459-465, September 1991.

[19] J. Stoer and R. Bulirsch, Introduction to numerical analysis, 2nd edition, Springer, New York, 1993.

[20] Eric Veach and Leonidas J. Guibas, "Optimally combining sampling techniques for monte carlo rendering", ACM Computer Graphics Proceedings, (ACM SIGGRAPH '95 Proceedings), pp. 419-428, 1995.

[21] G.J. Ward, F.M. Rubinstein, R.D. Clear, "A Ray Tracing Solution for Diffuse Interreflection", Computer Graphics (SIGGRAPH '88 Proc.), vol. 22, pp. 85-92, August 1988. 